\title{
Weyl Invariance of String Theories in Generalized Supergravity Backgrounds
}

\author{
José J. Fernández-Melgarejo, ${ }^{1, *}$ Jun-ichi Sakamoto, ${ }^{2, \dagger}$ Yuho Sakatani, ${ }^{3, *}$ and Kentaroh Yoshida, ${ }^{2, \S}$ \\ ${ }^{1}$ Departamento de Física, Universidad de Murcia, Campus de Espinardo E-30100, Murcia, Spain \\ ${ }^{2}$ Department of Physics, Kyoto University, Kyoto 606-8502, Japan \\ ${ }^{3}$ Department of Physics, Kyoto Prefectural University of Medicine, Kyoto 606-0823, Japan
}

(Received 23 December 2018; published 21 March 2019)

\begin{abstract}
We revisit Weyl invariance string theories in generalized supergravity backgrounds. A possible counterterm was constructed in a work by Sakamoto, Sakatani, and Yoshida, but it seems to be a point of controversy in some literatures whether or not it is nonlocal. To settle down this issue, we show that the counterterm may be local and exactly cancels out the one-loop trace anomaly in generalized supergravity backgrounds.
\end{abstract}

DOI: 10.1103/PhysRevLett.122.111602

Introduction.-A great progress in the recent study of string theory is that the generalized supergravity equations of motion (GSE) [1-3] (historically, GSE were discovered in the study of Yang-Baxter deformations of the $\mathrm{AdS}_{5} \times \mathrm{S}^{5}$ superstring [4,5], though the bosonic part has already appeared in much older literature [6]) have been derived from the $\kappa$-symmetry constraints in the Green-Schwarz (GS) formulation of superstring theories [3]. It is well known that the usual supergravity equations of motion are solutions to the $\kappa$-symmetry constraints $[7,8]$, but the discovery of this new supergravity indicates that there might exist more generalized supergravities.

In this Letter, we are concerned with string theory defined on generalized supergravity backgrounds (i.e., solutions to GSE). As a remarkable characteristic of GSE, a nondynamical vector field $I$ is contained. In order to solve the $\kappa$-symmetry constraints, it should be a Killing vector, and this Killing condition plays a crucial role in our later discussion. It is instructive to note that this Killing condition was not taken into account in the old literature [6,9], where a prototype of GSE was derived from the oneloop finiteness (or the scale invariance) of string theory. In addition, this extra vector field may be identified with the trace of nongeometric $Q$ flux, and many solutions of GSE can be regarded as $T$ folds [10].

There is an issue with the consistency of string theories in generalized supergravity backgrounds. As a matter of course, at a classical level, there is no problem. Thanks to other work [3], the $\kappa$ symmetry is ensured in generalized supergravity backgrounds and the GS formulation is consistently available. The issue arises at a quantum level.

Published by the American Physical Society under the terms of the Creative Commons Attribution 4.0 International license. Further distribution of this work must maintain attribution to the author(s) and the published article's title, journal citation, and DOI. Funded by SCOAP ${ }^{3}$.
Indeed, the Weyl anomaly may appear in string theories on generalized supergravity backgrounds $[2,6]$. In a recent work [1], Weyl invariance of bosonic string theories on generalized supergravity backgrounds was shown by constructing a possible counterterm \{this counterterm was inspired from the embedding of GSE into double field theory (DFT) [1]. For the detail of the notation, see Ref. [1]\} as

$$
S_{\mathrm{FT}}=\frac{1}{4 \pi} \int d^{2} \sigma \sqrt{-\gamma} R^{(2)} \Phi_{*}, \quad \Phi_{*} \equiv \Phi+I^{i} \tilde{Y}_{i} .
$$

This is a generalization of the standard coupling to dilaton $\Phi$, the so-called Fradkin-Tseytlin (FT) term [11]: indeed the standard FT term is reproduced when $I^{i}=0$. To be more concrete, in generalized supergravity backgrounds, the Weyl anomaly takes the following form

$$
\left\langle T_{a}^{a}\right\rangle=-\mathcal{D}_{a}\left[\left(Z_{m} \gamma^{a b}-I_{m} \varepsilon^{a b}\right) \partial_{b} X^{m}\right],
$$

which is canceled out introducing the counterterm [Eq. (1)]. Compared to the sigma model action, the counterterm [Eq. (1)] is higher order in $\alpha^{\prime}$, and it should be regarded as a quantum correction. Note also that the Killing vector $I$ entering the GSE, does not appear in the classical action of string sigma model, but first appears as a quantum correction at a stringy level.

A point of controversy in some literature [12-18] is whether the counterterm [Eq. (1)] is local or not. The integrand depends on the dual coordinate $\tilde{Y}_{i}$. In computing its contribution to the trace of the energy-momentum tensor $T^{a}{ }_{a}$, we need to use the equation of motion of the double sigma model $[19,20]$,

$$
\partial_{a} \tilde{Y}_{i}=g_{\mathrm{in}} \varepsilon_{a}^{b} \partial_{b} X^{n}+B_{\mathrm{in}} \partial_{a} X^{n} .
$$

This equation implies that $\tilde{Y}_{i}$ would be a nonlocal function of $X^{m}$ and one may suspect that the counterterm [Eq. (1)] is 
nonlocal as well. However, as we show in this Letter, we can construct a possible local counterterm by taking account of the fact that the two-dimensional Ricci scalar $R^{(2)}$ is locally a total derivative (we really appreciate J. Maldacena for elucidating this point) and $I$ is a Killing vector. That is, the (possible) nonlocality in the integrand of [Eq. (1)] can be removed. This is the main claim in this Letter.

Weyl invariance of bosonic string.-Let us first recall the basics on Weyl invariance of bosonic string theory in $D=26$ dimensions,

$$
S_{b}=-\frac{1}{4 \pi \alpha^{\prime}} \int d^{2} \sigma \sqrt{-\gamma}\left(g_{m n} \gamma^{a b}-B_{m n} \varepsilon^{a b}\right) \partial_{a} X^{m} \partial_{b} X^{n} .
$$

The Weyl anomaly of this system takes the form,

$$
2 \alpha^{\prime}\left\langle T_{a}^{a}{ }_{a}\right\rangle=\left(\beta_{m n}^{g} \gamma^{a b}-\beta_{m n}^{B} \varepsilon^{a b}\right) \partial_{a} X^{m} \partial_{b} X^{n} .
$$

Here, the $\beta$ functions at the one-loop level have been computed (e.g., in Ref. [6]) as

$$
\begin{aligned}
& \beta_{m n}^{g}=\alpha^{\prime}\left(R_{m n}-\frac{1}{4} H_{m p q} H_{n}{ }^{p q}\right), \\
& \beta_{m n}^{B}=\alpha^{\prime}\left(-\frac{1}{2} D^{k} H_{k m n}\right),
\end{aligned}
$$

where $D_{m}$ and $R_{m n}$ are the covariant derivative and the Ricci tensor associated with the spacetime metric $g_{m n}$ and $H_{m n p} \equiv 3 \partial_{[m} B_{n p]}$. For the Weyl invariance of the worldsheet theory, it is not necessary to require $\beta_{m n}^{g}=\beta_{m n}^{B}=0$. As long as they take the form

$$
\beta_{m n}^{g}=-2 \alpha^{\prime} D_{m} \partial_{n} \Phi, \quad \beta_{m n}^{B}=-\alpha^{\prime} \partial_{k} \Phi H^{k}{ }_{m n},
$$

the Weyl anomaly has a simple form

$$
\left\langle T^{a}{ }_{a}\right\rangle \stackrel{\text { e.o.m. }}{\sim}-\mathcal{D}^{a} \partial_{a} \Phi
$$

under the equations of motion. Here, $\mathcal{D}_{a}$ is the covariant

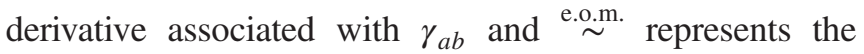
equality up to the equations of motion. This anomaly can be canceled out by adding the FT term [11],

$$
S_{\mathrm{FT}}=\frac{1}{4 \pi} \int \mathrm{d}^{2} \sigma \sqrt{-\gamma} R^{(2)} \Phi .
$$

Therefore, as long as the target space satisfies Eq. (6), namely the supergravity equations of motion,

$$
\begin{aligned}
R_{m n}-\frac{1}{4} H_{m p q} H_{n}{ }^{p q}+2 D_{m} \partial_{n} \Phi & =0, \\
-\frac{1}{2} D^{k} H_{k m n}+\partial_{k} \Phi H^{k}{ }_{m n} & =0,
\end{aligned}
$$

the Weyl invariance is ensured. As shown in Ref. [21], Eq. (9) implies that

$$
\beta^{\Phi} \equiv R+4 D^{m} \partial_{m} \Phi-4|\partial \Phi|^{2}-\frac{1}{12} H_{m n p} H^{m n p}
$$

is constant, and by choosing $\beta^{\Phi}=0$, we obtain the usual dilaton equation of motion.

The main observation of this Letter is that the requirement [Eq. (6)] is a sufficient condition for the Weyl invariance but is not necessary.

Local counterterm for GSE.-Let us consider a milder requirement,

$$
\begin{aligned}
& \beta_{m n}^{g}=-2 \alpha^{\prime} D_{(m} Z_{n)}, \\
& \beta_{m n}^{B}=-\alpha^{\prime}\left(Z^{k} H_{k m n}+2 D_{[m} I_{n]}\right),
\end{aligned}
$$

where $I_{m}$ and $Z_{m}$ are certain vector fields in the target space, which are functions of $X^{m}(\sigma)$. The condition [Eq. (11)] reduces to Eq. (6) when $Z_{m}=\partial_{m} \Phi$ and $I^{m}=0$.

Suppose here that $I_{m}$ and $Z_{m}$ satisfy

$$
\begin{aligned}
£_{I} g_{m n} & =0, & & I^{p} H_{p m n}+2 \partial_{[m} Z_{n]}=0, \\
\mathfrak{f}_{I} \Phi & =0, & & Z_{m} I^{m}=0 .
\end{aligned}
$$

In this case, the string sigma model has a conserved current associated with the global symmetry $X^{m} \rightarrow X^{m}+\epsilon I^{m}$, where $\epsilon$ is an infinitesimal constant. Then the on-shell conserved Noether current is given by

$$
J^{a} \equiv\left[I^{m}\left(g_{m n} \gamma^{a b}-B_{m n} \varepsilon^{a b}\right)-\tilde{I}_{n} \varepsilon^{a b}\right] \partial_{b} X^{n},
$$

where the 1 -form $\tilde{I}_{m}$ is defined through

$$
Z_{m}=\partial_{m} \Phi+I^{n} B_{n m}+\tilde{I}_{m} .
$$

When the $\beta$ functions take the forms of Eq. (11), the Weyl anomaly [Eq. (4)] becomes

$$
\left\langle T^{a}{ }_{a}\right\rangle \stackrel{\text { e.o.m. }}{\sim}-\mathcal{D}_{a}\left[\left(Z_{m} \gamma^{a b}-I_{m} \varepsilon^{a b}\right) \partial_{b} X^{m}\right] .
$$

Then, there is a rigid scale invariance [6], but it had been believed that the Weyl invariance would be broken because the counterterm [Eq. (8)] cannot cancel out the anomaly [Eq. (15)]. However, we will construct a modified local counterterm so as to cancel out Eq. (15).

Recall that the Lagrangian of the two-dimensional gravity is locally a total derivative,

$$
\sqrt{-\gamma} R^{(2)}=\partial_{a} \alpha^{a},
$$

where $\alpha^{a}$ is a vector density that should transform as

$$
\delta_{\xi} \alpha^{a}=£_{\xi} \alpha^{a}=\xi^{b} \partial_{b} \alpha^{a}-\alpha^{b} \partial_{b} \xi^{a}+\partial_{b} \xi^{b} \alpha^{a},
$$


under diffeomorphisms on the worldsheet. We then introduce the following counterterm (since $\alpha^{a}$ is defined only locally, the integral itself here should be defined more carefully depending on topologies of the string worldsheet)

$$
S_{\mathrm{FT}}^{(I, Z)}=-\frac{1}{4 \pi} \int d^{2} \sigma \alpha^{a}\left(Z_{m} \partial_{a} X^{m}-I_{m} \varepsilon_{a}{ }^{b} \partial_{b} X^{m}\right) .
$$

Note that this reduces to the FT term [Eq. (8)] when $I^{m}=0$ and $Z_{m}=\partial_{m} \Phi$. Supposing that $Z_{m}$ and $I_{m}$ are independent of $\gamma_{a b}$, the contribution of the counterterm [Eq. (18)] to the Weyl anomaly becomes

$$
\begin{aligned}
\langle T\rangle_{\mathrm{FT}}= & \frac{4 \pi}{\sqrt{-\gamma}} \gamma^{a b} \frac{\delta S_{\mathrm{FT}}^{(I, Z)}}{\delta \gamma^{a b}} \\
= & \mathcal{D}_{a}\left[\left(Z_{m} \gamma^{a b}-I_{m} \varepsilon^{a b}\right) \partial_{b} X^{m}\right] \\
& -\varphi^{a}{ }_{a} \mathcal{D}_{c}\left[\left(I_{m} \gamma^{c d}-Z_{m} \varepsilon^{c d}\right) \partial_{d} X^{m}\right] .
\end{aligned}
$$

Here, suggested by the identity in two dimensions,

$$
\delta\left(\sqrt{-\gamma} R^{(2)}\right)=\partial_{c}\left[\sqrt{-\gamma}\left(\gamma^{c a} \mathcal{D}^{b} \delta \gamma_{a b}-\gamma^{a b} \mathcal{D}^{c} \delta \gamma_{a b}\right)\right],
$$

we have used the variation

$$
\begin{aligned}
\delta \alpha^{c}= & \sqrt{-\gamma}\left(\gamma^{c a} \mathcal{D}^{b} \delta \gamma_{a b}-\gamma^{a b} \mathcal{D}^{c} \delta \gamma_{a b}\right) \\
& +\epsilon^{c d} \partial_{d}\left(\varphi^{a b} \delta \gamma_{a b}\right)
\end{aligned}
$$

where $\varphi^{a b}$ is a symmetric tensor made of the fundamental fields and their derivatives. In fact, the divergence in the last term of Eq. (19) vanishes by using the on-shell conservation law of a Noether current

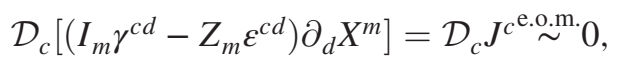

and we obtain

$$
\langle T\rangle_{\mathrm{FT}} \stackrel{\text { e.o.m. }}{\sim} \mathcal{D}_{a}\left[\left(Z_{m} \gamma^{a b}-I_{m} \varepsilon^{a b}\right) \partial_{b} X^{m}\right]
$$

Thus, this can exactly cancel out the anomaly [Eq. (15)].

Actually, the requirement [Eq. (11)] was proposed as the condition for the one-loop finiteness of string sigma model [6]. Now, we found that the Weyl symmetry can also be preserved upon introducing the above counterterm; hence one may anticipate that string theory should be consistently defined with the relaxed condition [Eq. (11)]. In the following, we will explain the condition [Eq. (11)] in terms of supergravity.

Generalized supergravity e.o.m.-From Eqs. (5) and (11), the condition for the Weyl invariance can be expressed as modified supergravity equations of motion,

$$
\begin{aligned}
R_{m n}-\frac{1}{4} H_{m p q} H_{n}{ }^{p q}+2 D_{(m} Z_{n)} & =0, \\
-\frac{1}{2} D^{k} H_{k m n}+Z^{k} H_{k m n}+2 D_{[m} I_{n]} & =0 .
\end{aligned}
$$

In fact, these are GSE for $g_{m n}$ and $B_{m n}$ originally proposed in Ref. [2] and later derived in Ref. [3] from the requirement for the $\kappa$ invariance of the GS type IIB superstring theory on an arbitrary background. There, the conditions [Eq. (12) ]are also required for the $\kappa$ invariance, and then equations of motion [Eq. (24)] lead to the following generalized dilaton equation of motion:

$$
R-\frac{1}{12}|H|^{2}+4 D_{m} Z^{m}-4\left(|I|^{2}+|Z|^{2}\right)=0 .
$$

Equations of motion in Eqs. (24) and (25) define the NS-NS sector of the generalized supergravity. See Refs. [1-3] for the modified equations of motion for the Ramond-Ramond fields. In particular, when $Z_{m}=\partial_{m} \Phi$ and $I^{m}=0$, these reduce to the conventional supergravity equations of motion.

In general, from the condition [Eq. (12)], we can choose a particular gauge where the 1 -form $\widetilde{I}_{m}$ in Eq. (14) vanishes $[1,2]$. Therefore, in the generalized supergravity, the generalization is characterized only by the vector field $I^{m}$. Note also that due to the presence of a Killing vector, any solution to GSE may be regarded as a nine-dimensional background via compactification on a circle.

In earlier works, many solutions to GSE have been obtained from the $q$ deformation [22], homogeneous YangBaxter deformations [10,23-25], and non-Abelian $T$ duality $[10,15,26]$ (see also Ref. [13]), while it was not clarified whether these solutions are consistent string backgrounds at a quantum level or not. However, the cancellation of the Weyl anomaly that we have explicitly shown here would be an important step towards clarifying the quantum consistency (the solution obtained from the $q$ deformation includes an imaginary Ramond-Ramond field, and would not be a consistent string background).

As presented in Refs. [1,27], we can regard solutions to GSE as solutions in DFT [28-31], which is a manifestly $T$-duality covariant formulation of supergravity. For the solutions of DFT, by using adapted coordinates where the Killing vector $I^{m}$ is constant, we find that the dilaton has a linear dependence on the dual coordinate $\tilde{x}_{m}[1]$. Moreover, if we perform a formal $T$ duality (a formal $T$ duality means the factorized $T$ duality along a nonisometry direction $x^{z}$, which maps the coordinate $x^{z}$ into the dual coordinate $\tilde{x}_{z}$. Such a transformation is a symmetry of the equations of motion of DFT) along the $I^{m}$ direction, an arbitrary solution to GSE is mapped to a solution of the conventional supergravity that has a linear coordinate dependence in the dilaton $[1,2,32]$.

Constructions of local $\alpha^{a}$.- So far, we have not presented an explicit form of the vector density $\alpha^{a}$. Let us 
explain here two ways to construct $\alpha^{a}$. Naïvely, from the defining relation,

$$
\sqrt{-\gamma} R^{(2)}=\partial_{a} \alpha^{a},
$$

one might expect that $\alpha^{a}$ can be expressed consistently in terms of the metric $\gamma_{a b}$. However, it is not the case as it is clearly explained in Refs. [33,34]. To construct $\alpha^{a}$ in terms of the metric $\gamma_{a b}$, the general covariance on the worldsheet should be broken. On the other hand, similarly to the approach of Ref. [33], if we introduce a zweibein $e_{\bar{a}}{ }^{a}$ on the worldsheet ( $\bar{a}$ and $\bar{b}$ are the flat indices), we find that

$$
\alpha^{a}=-2 \sqrt{-\gamma} e_{\bar{a}}^{a} \omega_{\bar{b}}^{\bar{b}} \bar{a}
$$

satisfies Eq. (26), where $\omega_{\bar{a}}{ }^{\bar{b}} \bar{c}$ is the spin connection. In this case, despite $\alpha^{a}$ being manifestly covariant under diffeomorphisms, it is not covariant under the local Lorentz symmetry. In the following, we will introduce two possible manners to construct covariant expressions of $\alpha^{a}$.

A construction with Noether current: The first approach is based on the approach explained in Sec. II. B of Ref. [34]. In two dimensions, if there exists a normalized vector field $n^{a}\left(\gamma_{a b} n^{a} n^{b}= \pm 1 \equiv \sigma\right)$, we can show

$$
\sqrt{-\gamma} R^{(2)}=2 \sigma \partial_{a}\left[\sqrt{-\gamma}\left(n^{b} \mathcal{D}_{b} n^{a}-n^{a} \mathcal{D}_{b} n^{b}\right)\right] .
$$

In string theories on generalized supergravity backgrounds, there exists a natural vector field on the worldsheet, which is the Noether current $J^{a}$ in Eq. (13). Supposing $J^{a}$ is not a null vector on the worldsheet, the vector field $n^{a}$ can be defined as $n^{a} \equiv\left(J^{a} / \sqrt{\sigma \gamma_{c d} J^{c} J^{d}}\right)$. Then $\alpha^{a}$ is defined as

$$
\alpha^{a} \equiv 2 \sigma \sqrt{-\gamma}\left(n^{b} \mathcal{D}_{b} n^{a}-n^{a} \mathcal{D}_{b} n^{b}\right),
$$

which is manifestly covariant and a local function of the fundamental fields. Moreover, by taking a variation of this $\alpha^{a}$ in terms of $\gamma_{a b}$, where the Noether current transforms as

$$
\delta\left(\sqrt{-\gamma} J^{a}\right)=\delta\left(\sqrt{-\gamma} \gamma^{a b}\right) \partial_{b} X^{m} I_{m},
$$

after a tedious computation, we find the desired variation formula [Eq. (21)] with $\varphi^{a b}$ given by

$$
\varphi^{a b}=\sigma\left(n^{c} \varepsilon_{c}{ }^{(a} n^{b)}+\frac{2 \varepsilon^{(a}{ }_{(c} \delta_{d)}^{b)}}{\sqrt{\sigma \gamma_{g h} J^{g} J^{h}}} \mathcal{D}^{c} X^{m} I_{m} n^{d}\right) .
$$

Therefore, this fully determines the variation of $\alpha^{a}$, for which the Weyl anomaly is canceled out in generalized supergravity backgrounds.

A construction from a gauged sigma model: As the second approach, we shall introduce some auxiliary fields to construct $\alpha^{a}$. For simplicity, we take a gauge $\tilde{I}_{m}=0$ here.
Let us consider the action of a gauged sigma model

$$
\begin{aligned}
S^{\prime}= & -\frac{1}{4 \pi \alpha^{\prime}} \int d^{2} \sigma \sqrt{-\gamma}\left[\left(g_{m n} \gamma^{a b}-B_{m n} \varepsilon^{a b}\right) D_{a} X^{m} D_{b} X^{n}\right. \\
& \left.-\tilde{Z} \varepsilon^{a b} F_{a b}\right],
\end{aligned}
$$

where $D_{a} X^{m} \equiv \partial_{a} X^{m}-I^{m} A_{a}, \quad F_{a b} \equiv \partial_{a} A_{b}-\partial_{b} A_{a}$, and $I \equiv I^{m} \partial_{m}$ satisfies the Killing equations. This model has a local symmetry,

$$
X^{m} \rightarrow X^{m}+I^{m} v, \quad A_{a} \rightarrow A_{a}+\partial_{a} v .
$$

This action can reproduce the bosonic string action $S_{b}$ after integrating out the auxiliary field $\tilde{Z}$. In order to cancel out the one-loop Weyl anomaly, we have to add the following local term to $S^{\prime}$ :

$$
S_{c} \equiv \frac{1}{4 \pi} \int d^{2} \sigma \sqrt{-\gamma} R^{(2)}(\Phi+\tilde{Z}),
$$

which is a higher order in $\alpha^{\prime}$. The contribution to the trace of the energy-momentum tensor coming from $S_{c}$ is

$$
\langle T\rangle_{c}=\frac{4 \pi}{\sqrt{-\gamma}} \gamma^{a b} \frac{\delta S_{c}}{\delta \gamma^{a b}} \stackrel{\text { e.o.m. }}{\sim} \mathcal{D}^{a}\left(\partial_{a} \Phi+\partial_{a} \tilde{Z}\right) .
$$

The equations of motion for $A_{a}$ and $\tilde{Z}$ give

$\partial_{a} \tilde{Z}=\varepsilon^{b}{ }_{a} J_{b}-|I|^{2} \varepsilon^{b}{ }_{a} A_{b}, \quad \varepsilon^{a b} F_{a b}=-\alpha^{\prime} R^{(2)}$,

where $J_{a}$ is the Noether current defined in Eq. (13). Since the field strength $F_{a b}$ vanishes to the leading order in $\alpha^{\prime}$, by using the local symmetry [Eq. (33)], we can find a gauge where the order $\mathcal{O}\left(\alpha^{\prime 0}\right)$ term vanishes

$A_{a}=0+\alpha^{\prime} \mathcal{A}_{a}, \quad \varepsilon^{a b}\left(\partial_{a} \mathcal{A}_{b}-\partial_{b} \mathcal{A}_{a}\right)=-R^{(2)}$.

Here, $\mathcal{A}_{a}$ is a quantity of order $\mathcal{O}\left(\alpha^{0}\right)$. Then the trace [Eq. (35)] is evaluated as

$$
\begin{aligned}
\langle T\rangle_{c} & \stackrel{\text { e.o.m. }}{\sim} \mathcal{D}^{a}\left(\partial_{a} \Phi+\varepsilon^{b}{ }_{a} J_{b}\right)+\mathcal{O}\left(\alpha^{\prime}\right) \\
& =\mathcal{D}_{a}\left[\left(Z_{m} \gamma^{a b}-I_{m} \varepsilon^{a b}\right) \partial_{b} X^{m}\right]+\mathcal{O}\left(\alpha^{\prime}\right) .
\end{aligned}
$$

This completely cancels out the one-loop Weyl anomaly [Eq. (15)], which comes from $S^{\prime}$.

After eliminating $\tilde{Z}$, the action $S^{\prime}+S_{c}$ becomes

$$
\begin{aligned}
S^{\prime}+S_{c}= & S_{b}+\frac{1}{4 \pi} \int d^{2} \sigma \sqrt{-\gamma}\left[R^{(2)} \Phi+\epsilon_{a b}\left(-2 \epsilon^{a c} \mathcal{A}_{c}\right) J^{b}\right. \\
& \left.-\alpha^{\prime}|I|^{2} \gamma^{a b} \mathcal{A}_{a} \mathcal{A}_{b}\right]
\end{aligned}
$$

As it is clear from Eq. (37), the gauge field $\mathcal{A}_{a}$ may be regarded as the desired $\alpha^{a}$ via $\alpha^{a}=-2 \epsilon^{a b} \mathcal{A}_{b}$. Then, by neglecting the higher order term in $\alpha^{\prime}$, the resulting 
expression is precisely the same as the standard sigma model action including our local counterterm $S_{\mathrm{FT}}^{(I, Z)}(18)$.

Note that the second term in the action [Eq. (39)] is the same as Eq. (5.13) of Ref. [16]. There, it was obtained by rewriting the nonlocal piece of the effective action $S_{\text {nonlocal }}$ of Ref. [12] through the identifications of $I_{m}$ and $Z_{m}$ with some quantities in the Yang-Baxter sigma model. In Ref. [12], the nonlocal action $S_{\text {nonlocal }}$ appeared in the process of non-Abelian $T$ duality, and it played an important role to show the tracelessness of $T_{a b}$. However, according to the nonlocal nature of the effective action, by truncating the nonlinear term by hand, it was concluded in Ref. [12] that the string model (called the B' model) is scale invariant but not Weyl invariant. On the other hand, the action [Eq. (39)] or our local counterterm [Eq. (18)] with $\alpha^{a}$ defined as Eq. (29) is local and free from the Weyl anomaly.

Conclusion and Discussion.-We have constructed a local counterterm [Eq. (18)] that cancels out the Weyl anomaly of bosonic string theory defined in generalized supergravity backgrounds, without introducing a manifestly $T$-duality-covariant formulation of string theory. This result supports the Weyl invariance of string theory in generalized supergravity backgrounds. In order to claim the quantum consistency of string theory in generalized supergravity backgrounds, it may be necessary to study some aspects of the associated CFT picture in more detail (e.g., higher genus cases), but the first nontrivial test has been passed. Here, we have considered the bosonic string theory, but the same counterterm should work in the RNS superstring theory as well.

Our result indicates new possibilities of string theory in more general backgrounds. In fact, if we appropriately choose the parameters of the nine-dimensional gauged supergravity $[35,36]$ and perform a formal $T$ duality along the ten-dimensional direction, we can obtain the GSE [37]. In DFT or its extension, the exceptional field theory, we can construct various deformed supergravities that are similar to GSE by performing the formal $T$ dualities and $S$ dualities [38]. It is important to study the consistency of string theories defined on solutions of these deformed supergravities. A reasonable conjecture is that as long as the target space satisfies the equations of motion of the exceptional field theory, the string theory could be defined consistently. We hope to come back on this interesting topic in our future researches.

K. Y. is very grateful to S. Iso, J. Maldacena, J.-H. Park, P. Townsend, A. Tseytlin, and K. Zarembo for valuable comments and discussions. Discussions during "11th Taiwan String Workshop," the workshop YITP-T-18-04 "New Frontiers in String Theory," and "The 5th Conference of the Polish Society on Relativity" were useful to complete this work. J. J. F.-M. acknowledges financial support of Fundación Séneca/Universidad de Murcia (Programa
Saavedra Fajardo). The work of J.S. is supported by the Japan Society for the Promotion of Science (JSPS). The work of Y.S. is supported by JSPS Grant-in-Aids for Scientific Research (C) 18K13540 and (B) 18H01214. The work of K. Y. is supported by the Supporting Program for Interaction based Initiative Team Studies (SPIRITS) from Kyoto University and by a JSPS Grant-in-Aid for Scientific Research (B) No. 18H01214. This work is also supported in part by the JSPS Japan-Russia Research Cooperative Program.

\section{*jj.fernandezmelgarejo@um.es \\ sakajun@gauge.scphys.kyoto-u.ac.jp \\ †yuho@koto.kpu-m.ac.jp \\ ${ }^{\S}$ kyoshida@gauge.scphys.kyoto-u.ac.jp}

[1] J. Sakamoto, Y. Sakatani, and K. Yoshida, Weyl invariance for generalized supergravity backgrounds from the doubled formalism, Prog. Theor. Exp. Phys. (2017), 053B07.

[2] G. Arutyunov, S. Frolov, B. Hoare, R. Roiban, and A. A. Tseytlin, Scale invariance of the $\eta$-deformed $\mathrm{AdS}_{5} \times \mathrm{S}^{5}$ superstring, T-duality and modified type II equations, Nucl. Phys. B903, 262 (2016).

[3] L. Wulff and A. A. Tseytlin, Kappa-symmetry of superstring sigma model and generalized 10d supergravity equations, J. High Energy Phys. 06 (2016) 174.

[4] F. Delduc, M. Magro, and B. Vicedo, An Integrable Deformation of the $\mathrm{AdS}_{5} \times \mathrm{S}^{5}$ Superstring Action, Phys. Rev. Lett. 112, 051601 (2014).

[5] I. Kawaguchi, T. Matsumoto, and K. Yoshida, Jordanian deformations of the $\mathrm{AdS}_{5} \times \mathrm{S}^{5}$ superstring, J. High Energy Phys. 04 (2014) 153.

[6] C. M. Hull and P. K. Townsend, Finiteness and conformal invariance in nonlinear $\sigma$ models, Nucl. Phys. B274, 349 (1986).

[7] M. T. Grisaru, P. S. Howe, L. Mezincescu, B. Nilsson, and P. K. Townsend, $N=2$ superstrings in a supergravity background, Phys. Lett. 162B, 116 (1985).

[8] E. Bergshoeff, E. Sezgin, and P. K. Townsend, Superstring actions in $D=3,4,6,10$ curved superspace, Phys. Lett. 169B, 191 (1986).

[9] J. Polchinski, Scale and conformal invariance in quantum field theory, Nucl. Phys. B303, 226 (1988).

[10] J. J. Fernandez-Melgarejo, J. Sakamoto, Y. Sakatani, and K. Yoshida, $T$-folds from Yang-Baxter deformations, J. High Energy Phys. 12 (2017) 108.

[11] E. S. Fradkin and A. A. Tseytlin, Effective field theory from quantized strings, Phys. Lett. 158B, 316 (1985).

[12] S. Elitzur, A. Giveon, E. Rabinovici, A. Schwimmer, and G. Veneziano, Remarks on nonAbelian duality, Nucl. Phys. B435, 147 (1995).

[13] B. Hoare and A. A. Tseytlin, Homogeneous Yang-Baxter deformations as non-abelian duals of the $\mathrm{AdS}_{5}$ sigmamodel, J. Phys. A 49, 494001 (2016).

[14] B. Hoare and F. K. Seibold, Poisson-Lie duals of the $\eta$ deformed symmetric space sigma model, J. High Energy Phys. 11 (2017) 014. 
[15] M. Hong, Y. Kim, and E. Ó Colgáin, On non-Abelian Tduality for non-semisimple groups, Eur. Phys. J. C 78, 1025 (2018).

[16] L. Wulff, Trivial solutions of generalized supergravity vs non-abelian T-duality anomaly, Phys. Lett. B 781, 417 (2018).

[17] R. Borsato and L. Wulff, Non-abelian T-duality and YangBaxter deformations of Green-Schwarz strings, J. High Energy Phys. 08 (2018) 027.

[18] B. Hoare and F. K. Seibold, Supergravity backgrounds of the eta-deformed $\mathrm{AdS}_{2} \times \mathrm{S}^{2} \times \mathrm{T}^{6}$ and $\mathrm{AdS}_{5} \times \mathrm{S}^{5}$ superstrings, J. High Energy Phys. 01 (2019) 125.

[19] M. J. Duff, Duality rotations in string theory, Nucl. Phys. B335, 610 (1990).

[20] C. M. Hull, A geometry for non-geometric string backgrounds, J. High Energy Phys. 10 (2005) 065.

[21] C. G. Callan, Jr., E. J. Martinec, M. J. Perry, and D. Friedan, Strings in background fields, Nucl. Phys. B262, 593 (1985).

[22] G. Arutyunov, R. Borsato, and S. Frolov, Puzzles of $\eta$ deformed $\mathrm{AdS}_{5} \times \mathrm{S}^{5}$, J. High Energy Phys. 12 (2015) 049.

[23] H. Kyono and K. Yoshida, Supercoset construction of YangBaxter deformed $\mathrm{AdS}_{5} \times \mathrm{S}^{5}$ backgrounds, Prog. Theor. Exp. Phys. (2016), 083B03.

[24] D. Orlando, S. Reffert, J. Sakamoto, and K. Yoshida, Generalized type IIB supergravity equations and nonAbelian classical r-matrices, J. Phys. A 49, 445403 (2016).

[25] J. Sakamoto and Y. Sakatani, Local $\beta$-deformations and Yang-Baxter sigma model, J. High Energy Phys. 06 (2018) 147.

[26] M. Gasperini, R. Ricci, and G. Veneziano, A problem with nonAbelian duality?, Phys. Lett. B 319, 438 (1993).
[27] Y. Sakatani, S. Uehara, and K. Yoshida, Generalized gravity from modified DFT, J. High Energy Phys. 04 (2017) 123.

[28] W. Siegel, Two vierbein formalism for string inspired axionic gravity, Phys. Rev. D 47, 5453 (1993).

[29] W. Siegel, Superspace duality in low-energy superstrings, Phys. Rev. D 48, 2826 (1993).

[30] C. Hull and B. Zwiebach, Double field theory, J. High Energy Phys. 09 (2009) 099.

[31] O. Hohm, C. Hull, and B. Zwiebach, Generalized metric formulation of double field theory, J. High Energy Phys. 08 (2010) 008.

[32] B. Hoare and A. A. Tseytlin, Type IIB supergravity solution for the T-dual of the $\eta$-deformed $\mathrm{AdS}_{5} \times \mathrm{S}^{5}$ superstring, J. High Energy Phys. 10 (2015) 060.

[33] S. Deser and R. Jackiw, Energy momentum tensor improvements in two-dimensions, Int. J. Mod. Phys. B 10, 1499 (1996).

[34] A. Yale and T. Padmanabhan, Structure of LanczosLovelock Lagrangians in critical dimensions, Gen. Relativ. Gravit. 43, 1549 (2011).

[35] E. Bergshoeff, T. de Wit, U. Gran, R. Linares, and D. Roest, (Non)Abelian gauged supergravities in nine-dimensions, J. High Energy Phys. 10 (2002) 061.

[36] J. J. Fernandez-Melgarejo, T. Ortin, and E. Torrente-Lujan, The general gaugings of maximal $d=9$ supergravity, J. High Energy Phys. 10 (2011) 068.

[37] See Supplemental Material at http://link.aps.org/ supplemental/10.1103/PhysRevLett.122.111602 for a proof.

[38] J. J. Fernández-Melgarejo, T. Kimura, and Y. Sakatani, Weaving the exotic Web, J. High Energy Phys. 09 (2018) 072. 